\title{
RECUPERAR EL DOLOR POR LA VÍA PLATÓNICA
}

\section{RECOVERING PAIN THROUGH THE PLATONIC WAY}

\author{
Luis A. Fallas López ${ }^{1}$ \\ Universidad de Costa Rica \\ luis.fallaslopez@ucr.ac.cr
}

Publicado 2016

\section{Resumen}

Este trabajo se dedica a representar los conceptos y las distintas formas de manejo del problema del dolor ${ }^{2}$ en Platón. Se tratan pasajes fundamentales de Protágoras, Fedón, Filebo, Timeo y Leyes. Atender al dolor terapéuticamente pareciera lo primario en los textos, ya que se constituye como un mal que debe ser resuelto, pero, además, como un fenómeno inevitable en la vida que puede llevarla a su propia destrucción. No obstante, la alternativa de extirpar tal afección no parece posible en la mayoría de los casos, incluso aunque tengamos un arsenal de herramientas racionales para contenerla; más bien, la estrategia sería recuperar nuestra capacidad para medir emociones, aquilatar su presencia y, como cuando nos rascamos, tratar de convertirlo en un factor a nuestro favor. Frente a nuestra contemporánea tendencia a extirpar todo dolor de nuestra vida, Platón, sobre todo el de las últimas obras, nos lleva a pensar, curiosamente, en la posibilidad de recuperarlo para que nos permita construir un camino más seguro hacia un bienestar integral en nuestra vida.

Palabras clave: dolor, ética, emociones, moderación, armonía, Platón.

1 El autor es doctor en Filosofía por la Universidad de Granada, España, catedrático de la Universidad de Costa Rica, docente en la Escuela de Filosofía de la Universidad de Costa Rica, en el Posgrado en Filosofía y diversas maestrías, así como en la Maestría Interuniversitaria de Bioética (Universidad Nacional y Universidad de Costa Rica). Ha sido profesor invitado y conferencista en varias universidades de Argentina, Perú, Chile, Brasil, España, Puerto Rico y México. Es coautor de En diálogo con los griegos (Bogotá, 2003), autor de Al acecho de lo puro. Introducción a una filosofía de lo singular en Platón (Bogotá, 2011) y de Emociones y bioética miradas desde la filosofía antigua (EUCR, 2015). Además, ha publicado alrededor de 40 artículos especializados en filosofía griega y ética en revistas de Brasil, Colombia, Chile y Costa Rica.

2 La traducción al español de todas las palabras escritas en griego no es posible por una razón básica: hay muchos sinónimos en griego para hablar del dolor, y esto es totalmente contextual. Odynē y lypē, por ejemplo, son ambas dolor, sus variaciones semánticas son amplias, pero no se podría ofrecer una sola traducción sin dejarse mucho por fuera. 


\section{Summary}

This paper is dedicated to represent the concepts and the various forms, in which the problem of pain is dealt with in Plato's work. Fundamental passages of Protagoras, Phaedo, Philebus, Timaeus and Laws are discussed. Attending to pain therapeutically seems to be the primary issue in these texts, as it is constituted as an evil that has to be resolved and, additionally, as an inevitable phenomenon of life that can also take it to its own destruction. However, the alternative of extracting the affliction does not seem to be possible in most cases, even if we have an arsenal of rational tools to contain it; rather, the strategy would be to regain our capacity to measure emotions, examine their presence and, in the same way we do when we scratch, turning them into a factor in our favor. Facing our contemporary tendency to extract every pain from our life, Plato, especially in his last works, leads us, curiously enough, to think about the possibility of regaining it, so it will allow us to build a safer path towards an integral wellbeing in our life.

Keywords: pain, ethics, emotions, moderation, harmony, Plato.

El dios, que quería reconciliar a esos (el placer y el dolor) que peleaban, dado que no lo logró, los ató por la coronilla a lo mismo; por eso, en lo que aparece uno, después va detrás el otro. (Fedón, 60c2-5)

Este mito que propone Sócrates al inicio del Fedón, que según el propio pensador bien lo podría haber compuesto Esopo, parece signar la dramática distinción entre su complicadísimo encuentro con Jantipa -obviamente marcado por el dolor y la desesperación de ella, al ver que ese mismo día él, su marido, habrá de morir-, y el que inicia con sus discípulos, un placentero encuentro lleno de emociones y razones. Además, nos mueve a pensar en la extraña resolución del maestro de Alópece de hacer caso, por fin, a un sueño repetido, y empezar a escribir poesía, precisamente en sus últimos días, cuando más bien parece necesario reforzar su purificación filosófica. Acaso podemos sumar todavía una relación de contrarios para considerar desde el mito: la que tendría la vida inmanente con la trascendente, dos hermanas que, para nuestros efectos, deberíamos suponer unidas también por la coronilla y que, en el diálogo, tendrán una importancia capital. Aunque de manera más directa y quizás casual, según el propio filósofo, se crea esta imagen para explicar la paradójica sensación que se tiene entre dolor y placer cuando uno se rasca. 
De hecho, le acaban de quitar los grillos con los que evitaban, en aque-

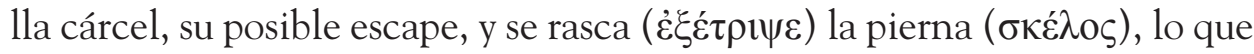
le hace reconocer la extrañeza del goce ${ }^{3}$, en un juego de contrariedad que maravilla (60b4), pues surge casi junto al dolor. Pero ni el goce ni el dolor quieren ( $\theta \dot{\lambda} \lambda \varepsilon \imath v)$ presentarse a un tiempo (b5-6), y más parecen encontrarse, inevitablemente, en algún momento o lugar que, según el mito, es en la parte más alta de su cabeza. En este sentido, si pensamos en el crecimiento importante de alguno de estos dos extremos (goce y placer), en paralelo se potencia su contrario, al punto de que, cuando uno está en su plenitud termina transformándose en el otro. Lejos estamos de lo que observa Aris-

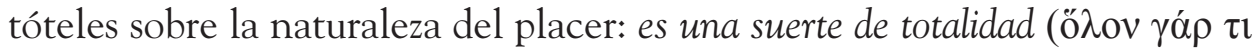

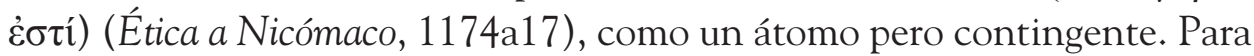
Sócrates esa totalidad se disiparía, transmutándose en su contraparte, aunque reaparecería casi sin querer, en un juego tan delicioso como aflictivo.

Así, Jantipa junto con sus emociones y realidades, que reflejan un dolor no querido, sin más, se aleja, se deja de preocupar, aunque exija hasta el máximo. Y de forma análoga, al llegar al final del encuentro filosófico, reaparecen las pasiones, esa angustia y dolor que no eran esperables en quienes estaban afianzados en la verdad, y eran capaces de discutir en el más alto nivel sin perder, por ello, la confianza. Y en el momento en que se debía manifestar esta última, en la corona del goce filosófico, aparecen las lágrimas y los gritos (117d), anticipando un final trágico cuando más bien era el paso definitivo a la plenitud. ¿Cómo no pensar que Sócrates esperaba coronar su paso por la inmanencia para llegar a la sabiduría propia de la plenitud de lo eidético, donde podríamos superar los estadios hipotéticos, aquel lugar donde ya no haría falta nada de lo anterior, para lo cual habría de mantener una pureza de mente y cuerpo que le permitiera acceder de una forma más consistente a lo que trasciende? Y, sin embargo, todo parece echarse a perder al final: el maestro no termina de convencer, los discípulos perdieron su dignidad y perspectiva, del placer de la palabra y el razonamiento pasaron al dolor de la sinrazón y los gemidos del desamparado.

3 Sobre el rascarse hay dos textos que merecen ser recordados: el primero, del Gorgias (494c6-8), en el que se habla de la felicidad propia del que sufre de sarna, que sería precisamente rascándose siempre -este ejemplo enfurece a Calicles, como es de esperar-. El segundo, del Filebo (46a8-9), donde se menciona el carácter terapéutico del rascado, obviamente para la picazón. 
Así pues, desde la alegoría socrática, es indudable que incluso para la mejor de las vidas posible las emociones son compañía necesaria, y en particular el dolor, pues con su gemelo a cuestas, el placer, tiene un papel preponderante, acaso definitorio de lo que somos los seres sintientes. Por supuesto, es más agradable y, quizás, esperable, tratar el problema del elemento placentero y positivo en esta dualidad, dado que es la cara buena y clara de estas complejas monedas que constituyen nuestras más fuertes emociones; pero lo que no gusta y tiene poca prensa quizás exige una mayor atención. Como dice Parménides, en el diálogo homónimo que le dedicara Platón: las cosas que despreciamos por poco filosóficas, como el pelo, la basura o el lodo, o para el caso que nos ocupa, el dolor, en el fondo han de exigirnos la misma atención que lo más sublime (130c-e), si es que nos dejamos atrapar por la filosofía (cf. e2).

No obstante, los lugares en que Platón trata los fenómenos de dolor son más bien escasos, quizás haciendo de contraparte a la tradición literaria que ha convertido la tragedia, las lamentaciones y demás expresiones de aflicción humanas en el modo de vida griego, con una suerte de fórmula imitativa que, como señala Aristóteles (Poética 1449b28), permite purgar las pasiones vividas. El filósofo ateniense opta claramente, y en mayor medida, por revalorar el poder de la racionalidad para medir, contener y, en algunos, suturar tales acontecimientos.

Aún así, es un hecho innegable que sucumbimos ante los dolores, y que, en muchos casos, estos determinan lo mejor que tenemos, acaso incluso más que los propios placeres. Por ello, es posible una reinserción en el problema, aprovechando sobre todo que el propio filósofo no ha dejado de darle el lugar que merece; aunque con esto vamos a romper la lógica de la lectura de los Diálogos, pues saltaremos de una época a la otra sin atender, mayormente, a la evolución que el autor mostró; a falta de un gran texto sobre la aflicción, no queda más que construir sus posibles alcances.

En el lenguaje de Platón, dolor puede decirse de varias maneras, dependiendo de intensidades y localizaciones. Las palabras más usuales para

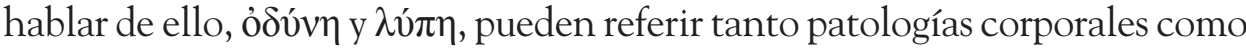
anímicas, aunque parecen describir mucho más el dolor físico. Para hablar 
de dolores fuertes e intensos, igualmente físicos, como los de parto, por

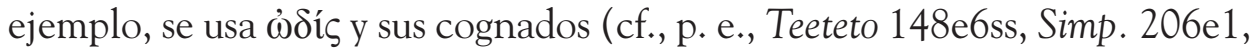
Fedro 251e5, Rep. 574a3 y 583d3, Leyes 873c5); mientras que la aflicción o el sufrimiento, que bien podrían verse más relacionados con sensaciones

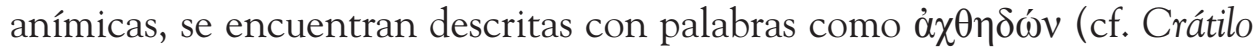
419c6, Leyes 734a6), àvía (cf. Prot. 351c, 353e, Crátilo 419c3, Gorg. 477d2)

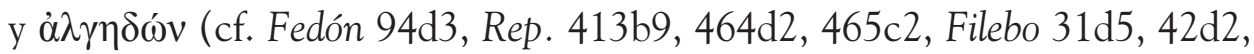
52a2, Leyes 647a5, etc.).

De esos términos, el que se utiliza un mayor número de veces es $\lambda v ́ \pi \eta$, quizás porque es el antónimo de ídoví (placer), y tal vez por la razón semán-

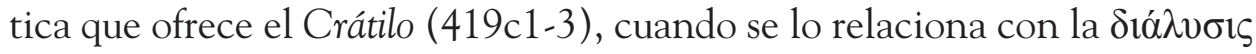
(disolución) corporal: este deterioro, o incluso destrucción, bien podría definir lo que se entiende por el dolor en general -según el mismo pasaje,

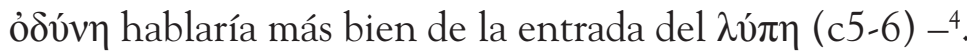

Esta visión del dolor, como una ruptura o pérdida de la naturaleza, se ve confirmada en la más clara y completa determinación de este fenómeno,

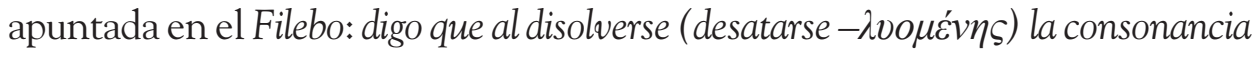
( $\dot{\alpha} \rho \mu o v i \alpha)$ en nosotros los seres vivos, a la vez en ese momento específico acontece una pérdida ( $\lambda v ́ \sigma v v)$ de la naturaleza y el nacimiento de los dolores ( $\dot{\alpha} \lambda \gamma \eta \delta o ́ v \omega v)$ (31d4-6). Nótese que la clave está en la ruptura de la "armonía" de las partes, como en un conjunto musical en el que se pierde la afinación y el ritmo, al punto de que ya no se muestra la identidad que marcaba al grupo; por eso ya no se es lo mismo, la constitución, la naturaleza del compuesto se rompe y surge el dolor. Si hablamos de una consonancia perdida, se asume que el ser sigue determinados procesos, movimientos, que son interrumpidos o desviados. En este sentido, recuerda el juego etimológico que describe el Crátilo al asumir que la $\dot{\alpha} \chi \theta \eta \delta \omega ́ v$ (pena o pesadumbre) es producto de -o corresponde a- un

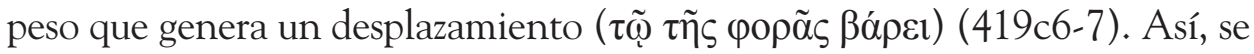
entenderá más adelante en el Filebo (43a), cuando se establece que grandes movimientos ( $\mu \varepsilon \tau \alpha \beta \mathrm{o} \lambda \alpha i$ ) son los que producen en nosotros dolores y placeres.

4 Para describir procesos de dolor severo se usa áviapó (cf. Prot. 351c-d, Gorg. 497d, Rep. 587e3), aunque quizás el sustantivo $\pi \varepsilon p 1 \omega \delta v v i ́ \alpha$ sea el que mejor signa esta condición (Rep. 383d3, Leyes 732c3 y 873c5). Para hablar del padecer se usa, por ejemplo, ódvvów (Prot. 351b5, Ión 539c5, Fedr. 251d6), y para signar el acto de infligir dolor están los verbos $\lambda v \pi \varepsilon ́ \omega$ y àvió $\omega$ (múltiples lugares). Un pasaje ha de tenerse en cuenta para las variables nominales: Filebo 42d1-3. Platón, probablemente, tiene presentes sutilezas en la distinción en esos sinónimos que no explicita. 
En el Timeo (64a-b) se sostiene una tesis parecida: primero queda claro que estos acontecimientos son pasiones $(\pi \alpha \theta \eta \dot{\mu} \mu \alpha \alpha)$, lo cual está en clara correspondencia con las percepciones, pero lo crucial es cómo se mueven, porque a mayor movilidad, mayor sensación. De esta manera, se da una especie de proceso mecánico entre las partes corporales -en forma circular (64b4-5) - que permite llevar la información hasta la inteligencia ( $\tau$ ò $\varphi \rho o ́ v i \mu o v)$, en la cual se conoce lo que lo ocasionó; por supuesto, en el caso de lo que se mueve poco, la percepción no se da o no resulta importante. Lo cierto es que es una pasión dolorosa ( $\pi \dot{\alpha} \theta$ os $\dot{\alpha} \lambda$ yevóv) la que nace en nosotros como un todo ( $\left.\dot{\alpha} \theta \rho o ́ o v{ }^{5}\right)$ contra la naturaleza y por la fuerza (Bíaıv) (c7-d1). En otras palabras, el acontecimiento es efectivamente doloroso cuando hay una ruptura de los procesos naturales que es violenta, y suficientemente fuerte como para que lo que está llamado a moverse lo haga al punto de llegar a las instancias receptoras, que por lo visto suponen un acto de conciencia -conocimiento racional, si atendemos al adjetivo $\varphi \rho o v u \mu o ́ s$ (inteligente) -. Esto no ocurre de la misma manera en todos los cuerpos ni partes; por ejemplo, en cuerpos compuestos por partes grandes es evidente que se afecta al conjunto, al generarse un movimiento contra su disposición natural (64e4-5). En cambio, en aquellos en los que las partes son muy pequeñas, como sería el caso de la vista, tal afectación no se daría (e3-4).

De cualquier manera, el dolor parece estar relacionado con elementos negativos, contra la naturaleza o males. Así lo da a entender el Sócrates del Protágoras, en unos pasajes en los cuales discute la difícil clasificación de algunos padecimientos que son dolorosos pero buscados por ser beneficiosos $\left(354 a^{6}\right)$. Lo que sí parece muy claro es que debería mantenerse más firme-

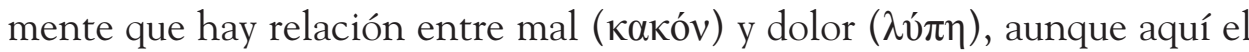
mal, quizás, se puede decir de varias maneras.

Mejor establecido está en el Gorgias, en el que se analoga con lo do-

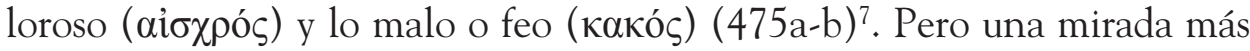
aclaradora se encuentra probablemente en el Filebo, en el cual no se afirma de forma contundente que se trata de algo malo, pero sí que su superación o

5 Atendiendo a este adjetivo, puede entenderse también como un evento repentino, que se da completo a un tiempo.

6 Los ejemplos que cita en este pasaje son perfectamente trasladables a nuestro mundo contemporáneo: los ejercicios gimnásticos, el servicio militar y los cuidados médicos.

7 Muñón (2013, p. 150) distingue algo que valdría recalcar, a partir de la discusión de Sócrates con Calicles en el Gorgias: entre placer y dolor hay una relación íntima, pero no así entre bien y mal, que no podrían coexistir. Esto nos permite, desde ya, apreciar que la correspondencia entre mal y dolor no podría ser de equiparación; de hecho el dolor será un instrumento por considerarse en múltiples sentidos, incluso para la formación de las personas. 
extirpación resulta un bien indudable para quien está enfermo (45b), esto es, para quien carece de salud manifestar una mejora genera bienestar, al punto de que se goza de un placer o un bien tal que se asume como el mayor posible.

No obstante, cabe la posibilidad de distanciar dolor y mal, como de hecho se hace en el Timeo, en el cual es un padecimiento que aleja del bien, pero no necesariamente provoca lo malo, cosa que sí logra de manera contundente el placer (69d). Aquí se habla del mal posiblemente en orden moral, aunque el pasaje refiere al alma mortal, la cual se ve llevada por esas "terribles y necesarias pasiones" (69c8-d1), y allí lo que pesa ha de ser el rigor de una moderación que no permite incorrecciones superiores.

La relación del dolor con lo malo, como lo suponen estos pasajes del Timeo, tiene más que ver con el grado de voluntariedad, y en este sentido es menos esperable que se busque de por sí. Frente a esto, su contraparte, el placer, sería un objeto al que cualquiera tendería. Aun así, es fundamental ver cuánto se acerca precisamente al placer, a fin de terminar el cuadro que lo enmarca. A este propósito debe reconocer que existe en los humanos

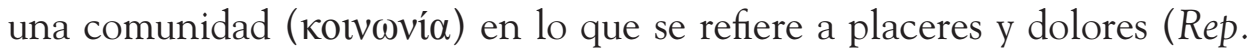
462b4), en el sentido de que marchan a un mismo nivel en la determinación de nuestras vidas: para mí en un género ( $\gamma \varepsilon ́ v \varepsilon ı)$ común al mismo tiempo parecen nacer el dolor y el placer conforme con la naturaleza (31c2-3). Esto hace pensar ya no en la imagen de los siameses del Fedón, planteada al inicio, sino en un conjunto de acontecimientos que, pareciendo contrarios, en realidad resultan paralelos e incluso llegan a confundirse, como lo muestra el ejemplo del rascarse, que duele y agrada, del propio Sócrates -esto se repite en el Filebo al hablar de una suerte de disposición ( $\left.\delta \imath^{\alpha} \theta \varepsilon \sigma ı \varsigma\right)$ en la que no se sabe, estrictamente si hay goce o aflicción (32e-33a) -. Esto es especialmente evidente en los espectáculos trágicos, en los que a un tiempo los que

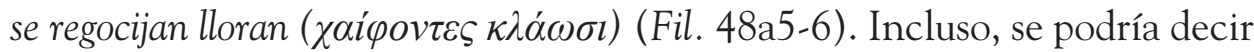
que ambos afectos se producen a la vez, como ocurre cuando nos reímos de ridiculeces de amigos nuestros, uniendo un dolor, la envidia y el gozo, y así en miles de otros casos (50b4). Estas ocasiones nos suelen agradar y, por eso, se buscan de manera particular cuando hablamos del teatro, donde, como espectadores, atendemos con gran reconocimiento a las virtudes de los artistas, por ser capaces de hacernos gozar de dolores maravillosamente representados (cf. Rep. 605c). 
De cualquier manera, sea ante dolores puros o mezclados, pareciera que lo fundamental está en cómo atiende nuestra alma el asunto, pues es ella la que es capaz de "poner atención" como el caso de la envidia (Fil. 48b) y tantas otras afectaciones, que se sufren al punto de quedar constreñido, como ver el mal de un amigo (49d) o la cólera, los celos, etc. $(47 \mathrm{e})^{9}$; o en algo más bien corporal, como pareciera lo común. La ruptura del orden natural exige un conocimiento, e incluso una cierta capacidad de manejo.

$$
* * *
$$

La vida correcta (ó $\rho \theta \dot{o} v)$ no ha de perseguir los placeres, ni tampoco huir del todo de los dolores ( $\lambda \dot{v} \pi \alpha \varsigma$ ), sino abrazar amablemente ( $\dot{\alpha} \sigma \pi \alpha ́ \zeta \varepsilon \sigma \theta \alpha l)$ el medio ( $\mu \varepsilon \dot{\sigma} \sigma o v)$ mismo, ese al que me he referido al nombrarlo como propicio ( $i \lambda \varepsilon \omega v)$, una disposición ( $\delta i \alpha ́ \theta \varepsilon \sigma l v)$ del dios a la que por algún mensaje mántico con acierto saludamos todos. (Leyes 792c9-d4)

Con el alma como ama y señora del cuerpo (cf. Fedón 94c10-d6), una conductora que incluso al tratar las penurias hace uso de terapias dolorosas ( $\left.\mu \varepsilon \tau^{\prime} \dot{\alpha} \lambda \gamma \eta \delta \delta^{\prime} \omega \omega\right)$, como las propias de la gimnasia y la medicina (94d3-4), debería ser posible sobrellevar o corregir las pasiones más amargas, de manera tal que al menos se comprendan y asimilen.

La estrategia ante el dolor está en la medida, como lo supone el Protágoras (cf. 256a1-5), aplicando la "metrética", el arte que mide el exceso y la deficiencia (357a1-2), que resulta de una extraordinaria importancia, pues asegura el saber y la propia subsistencia. Aunque en esto parece muy difícil hablar de mediciones estrictas, como supone el Filebo, porque son asuntos

8 Se puede atender a algo que está ocurriendo o también a algo por anticipado, como bien destaca Mié (2012, p. 168) a partir de Fil. 32c.

9 En 34b-35d se puede ver un tipo de dolor estrictamente anímico, el deseo, un evidente producto de una carencia de la que se hace uno consciente. Como interpreta Correa (2012), se podría decir que la carencia corporal es de segundo orden frente a la que tiene el alma, que es la que se da cuenta del padecimiento. Aunque pareciera obvio que el cuerpo, en la generalidad de los dolores, es el que realmente siente, como destaca Ganson a propósito del Timeo pero en cercanía con los pasajes que estudiamos del Filebo, "pains are housed in the mortal part of the soul $(65 \mathrm{a} 5$ and $69 \mathrm{~cd})$, but sensed affections are in the body, not the soul. For these reasons, I will assume that Plato takes pains and pleasures to be sensations whose objects are affections of the body" (2001, p. 229). 
que admiten "lo más" y "lo menos" (27e5-6). Es obvio que, ante tal problema, rozamos la indeterminabilidad, pues esto no implica que sea inmanejable: el asunto dejaría por fuera la exactitud aritmética para tender a las relaciones de proporcionalidad, siempre en busca de medir su dimensión ( $\mu \varepsilon \gamma \varepsilon \theta$ ó $\varsigma$ )

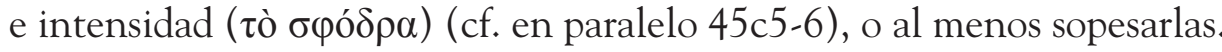

La balanza que nos permite pesar o comparar puede ser un mecanismo eficiente, pero habría que ver con qué elementos se habría de hacer la aquilatación. En principio, se procedería por la negación: dolor frente a su supresión, o comparación por fuerza, intensidad, lugar de manifestación, consecuencias, etc.; pero esto no nos permite una posición exclusiva, más cuando sabemos que el dolor pasa etapas que van de la mera presencia de una irritación

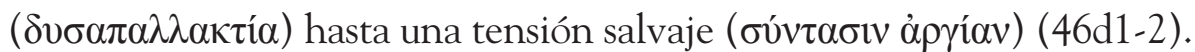

En cualquier caso, ante la desarmonización que ello significa, se hace necesario buscar una herramienta eficaz para controlar, de algún modo, el problema. Es en las Leyes donde la vemos clara, se trata de la siempre enaltecida $\sigma \omega \varphi \rho о \sigma u ́ v \eta$, esa excelencia capaz de atemperar excesos y que nos permite, más que eliminar sensaciones, pasiones y emociones, controlarlas, enfrentándolas pero con una prudente contención y no una ciega pretensión de extirpar, pues se volvería una lucha casi absurda, en la que, en general, se termina peor de lo que se empezó. Ella nos habrá de permitir un gobierno inteligente -prudente- de sí en perspectiva al bien (644b6-7).

Para esto, tenemos que conocer cuáles son los caminos errados, entre los cuales son dos malos informantes ( $\sigma 0 ́ \mu \beta 0 \lambda o \varsigma)$ el propio dolor, así como el placer (644c6-7), especialmente cuando se ven puros (799a3) y no somos capaces de buscar mediaciones (a4), pues terminan acompañados de emociones extremas, que nos desvinculan totalmente de las expectativas ciertas y adecuadas. En el caso del dolor, esto se refleja de manera muy clara, pues no hace sino ofrecernos la esperanza de mayores males; de ahí que, normalmente, nos llene de temor y, por consecuencia, nuble nuestro entendimiento: el

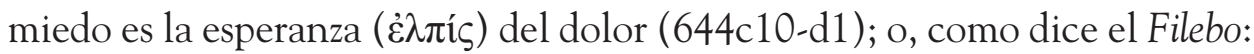
para los dolores es lo terrible (

Pero, lo crucial está en entender cómo se conforma nuestra alma: creamos que cada uno de nosotros los seres vivos somos como una marioneta divina, sea como un juguete para aquellos (los dioses) o como un constructo para algo útil; eso no lo sabemos, pero sí vemos esto: que esas pasiones en nosotros, como 
unos tendones o ciertas cuerdas internas, tiran de nosotros y siendo contrarias las unas a las otras nos llevan a acciones contrarias; desde lo cual en efecto resultan determinados la virtud y el vicio (Leyes 644d7-e4). Seamos o no dependientes de estos tendones, lo cierto es que, aún asumida nuestra fuerza interior, esas cuerdas que nos mueven habrían de producir tremendos desajustes, que nos son casi inevitables. Para colmo de males, no solo de un lado se tira, al otro extremo encontramos el placer y sus tentadoras ilusiones, y la misión está, precisamente, en resistir, aunque mejor que ello en comprender que, entre jalones y tropiezos, nos movemos, como una marioneta que bien ha de simular sus caídas, esforzándose por parecer aquello que busca imitar: una racional continencia que a nada se entrega completamente, pero tampoco renuncia del todo. Ha de recordarse que placeres y dolores son necesarios, definen lo que somos y sufrimos.

Hay que tener presente que entre estas cuerdas una exige un cuidado especial, pues no es de hierro, como esas pasiones descritas, sino de oro y, en consecuencia, es débil (645a2-3). Hablamos de la ley común de la ciudad, la conducción áurea y sagrada del razonamiento calculador, una cuerda a la que es necesario que cada uno siga sin desistir, pese a los embates de esos otros tendones (644e4-645a1).

Sabemos que, si el tendón del dolor se impone, termina reduciendo la capacidad crítica frente a lo que desfavorece la identidad propia, suspendiendo las vergüenzas que son aliadas naturales de nuestra dignidad: lágrimas, desvanecimientos, gritos, angustias, en general, asoman una humanidad que, comúnmente, no queremos reconocer, pero que ante lo doloroso acompañan, reconfortan y de algún modo alivian, pero desnudando.

Con todo, si atendemos a este Platón en plena vejez, lo mejor es buscar el equilibrio, esa música que respeta las diferencias y no se deja ahogar en los extremos, porque sabe medir, porque sabe que hay un ritmo vital que entona las armonías, el juego sensato que conjuga el movimiento con el reposo, lo agudo con lo bajo, sin que por ello estos dejen de estar allí, sonando y resonando ${ }^{10}$.

10 La música es, probablemente, la mejor manera de representar y regular la Magnesia de las Leyes y sus números: recordemos tan solo la división en partes de la ciudad, así como sus matemáticos repartos (cf. 745b-c), junto a lo cual vale traer a colación la importancia de lo musical en la formación educativa de la ciudad (cf. libro II). 


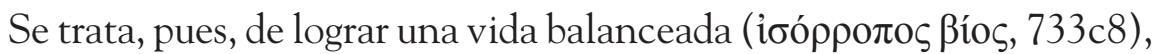
que sopese y apueste por lo mejor; a propósito de lo cual es quizás obvio que preferimos evitar dolores y promover placeres (733b), aunque a ambos acontecimientos nos encontramos atados por naturaleza ( $\dot{\varsigma} \xi \dot{\varepsilon} v \tau o v ́ \tau o r \varsigma$ $\left.\dot{\varepsilon} v \delta \varepsilon \delta \varepsilon \mu o^{\prime} v o 1 ~ \pi \varepsilon \varphi v ́ \kappa \alpha \sigma ı v, 733 \mathrm{~d} 2-3\right)$, por lo que parece necesario intentar sobrellevarlos, pese a las complejidades que supongan.

En los márgenes que nos permite la vida, se trata de aprender a elegir un balance entre lo que gusta y lo que no, lo que queremos y lo que se adecua a nuestro ser; lo cual muchas veces no deseamos, aunque no queda otra que prescribirlo. En este sentido, es esencial recordar que las variables son diversas, no solo en los actos específicos, sino también en los modos de vida en general.

La moderación permite que todo se vuelva más fácil de sobrellevar, de manera particular, por dirigirnos hacia dolores y placeres calmos ( (734a1); pero además logra una diferencia muy importante frente al incontinente ( $\dot{\alpha} \kappa o ́ \lambda \alpha \sigma \tau \sigma \varsigma)$, pues sus dolores son muy diferentes en su dimensión, número y frecuencia (734a7). De ahí que se pueda afirmar que ser incontinente no es algo ni deseable ni que realmente se quiera (734a4), habida cuenta de los sufrimientos que de ello se desprenden, aunque el populacho termina cayendo en estos excesos, sin duda producto de su debilidad, ignorancia o mala formación (734b5-6), y por ello merece un castigo importante (cf. 934a). En este sentido, es posible hablar de un manejo prudencial del dolor, tanto si se sufre sin buscarlo como si se realizan acciones que lo propicien, lo cual podría ser absurdo, pero resulta común.

Frente a ello, en un cierto lugar intermedio, podríamos reconsiderar la idea heraclítea de la necesidad de consideración entre contrarios, como la que puede mejor explicar el lugar que habríamos de darle a nuestros dolores, si usamos analógicamente el fragmento 111: la enfermedad hace placentera y buena la salud. Es decir, el dolor sería un potenciador de la vida, sobre todo de su riqueza, pues no es sino frente a él que empezamos a ser conscientes del bienestar que vivimos, de las posibilidades que desperdiciamos y, sobre todo, del complejo entramado que constituyen nuestros organismos: incluso si una muy pequeña parte se ve afectada, se percibe como si todo estuviese mal, y nuestra atención cambia radicalmente, centrándose en aquello que ha perdido su estado normal. 
Aunque, quizás, podríamos poner una mayor atención en el pitagorismo como una fuente que permite adecuar nuestra mirada sobre los padecimientos, poniendo énfasis en las mediaciones más que en las tensiones heraclíteas. Hablamos de las medias proporcionales que plantea el Timeo (36a), las cuales nos habrían de conceder encontrar lugares que podríamos considerar adecuados para una determinación estricta. Mediar pitagóricamente, eso sí desde una perspectiva platónica, significa encontrar lugares que permitan, en lo contingente y su indefinición, llegar a cierta formalidad estable, no con el valor o la seguridad que se supone tendrían las formas perfectas, pero sí con la consistencia necesaria para hablar de belleza y continuidad; así como ocurre con el mundo (cfr. Timeo 30a) y sus partes más sublimes (el cuerpo completo y su alma).

Dicho de otra manera, y cerca de la cuestión del padecimiento, podríamos reconstruir la idea de que, entre los extremos de una vida plena, sin mutación ni debilitamiento alguno, propia de los dioses, y la trágica, que supone el común griego, la cual renuncia a toda ilusión y determinación segura por estar siempre al filo de una navaja que tarde o temprano acabará con nuestras fortalezas e identidad, podemos edificar mediaciones proporcionales que nos den una continuidad básica, relacionada con una disposición que vamos constituyendo para asumir, con fortaleza, los acontecimientos más complicados que podamos encontrar en el camino. Con ello es posible que logremos esa vida correcta de la que habla el fragmento de Leyes con que iniciamos este apartado final, porque abrazados a un punto medio sabremos que huir de lo que nos incomoda, o incluso cercena y al final posiblemente nos llegue a destruir, quizás es no entender lo que significa nuestro propio bienestar.

\section{Referencias}

Aristotelis. (1962). Ethica nicomachea. Oxford: Clarendon Press.

Aristotelis. (1970). Poetica. Oxford: Clarendon Press.

Correa, A. (2012). Conocer y elegir el bien: Filebo 20b-22e. En Lozano-Vásquez, A., Ariza, S. y Correa, A. (Eds. y Colabs.), Platón y la irracionalidad. Bogotá: Universidad de los Andes (125-144).

Ganson, T. S. (2001). Appetitive Desire in Later Plato. History of Philosophy Quarterly, $18(3), 227-237$.

Laín Entralgo, P. (1958). La curación por la palabra en la antigüedad clásica. Madrid: Revista de Occidente. 
Le Breton, D. (1999). Antropología del dolor. Barcelona: Seix Barral.

Mié, F. (2012). Sobre las condiciones de la vida racional y afectiva humanas en el Filebo. En Lozano-Vásquez, A., Ariza, S. y Correa, A. (Eds. y Colabs.), Platón y la irracionalidad. Bogotá: Universidad de los Andes, (145-204).

Morris, D. (1994). What we make of pain. The Wilson Quarterly,18(4), 8-19.

Moscoso, J. (2011). Historia cultural del dolor. Madrid: Taurus.

Muñón Sáenz, A. R. (2013). El pensamiento de Platón a la luz de una nueva hermenéutica. Alicante: Editorial Club Universitario.

Platonis. (1967 [1900-1907]). Opera. Oxford: Clarendon Press. 